\title{
Reconstrucción del ligamento patelofemoral medial mediante suturas transóseas patelares: estudio transversal de 34 pacientes
}

\section{Reconstruction of the Medial Patellofemoral Ligament with Patellar Transosseous Sutures: Cross-Sectional Study with 34 patients}

\author{
Andrés Pakuts ${ }^{1,2(0)}$ Luis Fernando Martin ${ }^{3(0)}$ Christophe Riquoir ${ }^{4(-)}$ Alex Schadenberg 4
}

${ }^{1}$ Clínica Red Salud Elqui, La Serena, Chile

${ }^{2}$ Centro Médico Ossis, La Serena, Chile

${ }^{3}$ Residente Traumatología y Ortopedia, Facultad de Medicina Clínica

Alemana de Santiago-Universidad del Desarrollo, Santiago, Chile

${ }^{4}$ Interno, Facultad de Medicina, Pontificia Universidad Católica de Chile, Santiago, Chile

Rev Chil Ortop Traumatol 2021;62(2):e104-e112.
Address for correspondence Andrés Pakuts, Clínica Red Salud Elqui, La Serena, Chile (e-mail: andrespakuts@gmail.com).

\section{Resumen \\ Palabras clave \\ - ligamento patelofemoral medial \\ - reconstrucción \\ - inestabilidad rotuliana}

Introducción La inestabilidad rotuliana recurrente puede conducir a malos resultados funcionales y daño articular progresivo a largo plazo, y con frecuencia se aborda mediante la reconstrucción del ligamento patelofemoral medial (RLPFM), con múltiples técnicas que suelen diferir en el método de fijación de la rótula.

Objetivo Evaluar los resultados de RLPFM utilizando una técnica novedosa de fijación rotuliana mediante suturas transóseas. El objetivo principal es determinar las tasas de reluxación y los resultados funcionales incluyendo retorno deportivo. Los objetivos secundarios incluyen la evaluación de las complicaciones y de otras variables relacionadas, y la comparación entre los resultados de RLPFM aislada y asociada a una osteotomía de la tuberosidad tibial.

Métodos Estudio transversal de 34 pacientes sometidos a RLPFM desde 2013 hasta 2019 con un seguimiento mínimo de 12 meses. La reconstrucción fue realizada por el mismo primer cirujano con autoinjerto de gracilis de doble banda en todos los casos. La fijación del injerto en la cara medial de la rótula se realizó utilizando dos puntos de fijación transóseos independientes con suturas de alta resistencia, y la fijación femoral anatómica, con un tornillo interferencial mediante referencias anatómicas y radiológicas. Hubo 27 pacientes con RLPFM aislada, y 7 con osteotomía de la tuberosidad tibial asociada.

Resultados La edad media fue de 22,8 años (desviación estándar [DE]: 9,1). El 50\% eran hombres. El seguimiento desde la cirugía hasta el cuestionario fue de 30,4 meses recibido

17 de mayo de 2020

aceptado

22 de marcha de 2021
DOI https://doi.org/

$10.1055 / \mathrm{s}-0041-1735603$. ISSN $0716-4548$.

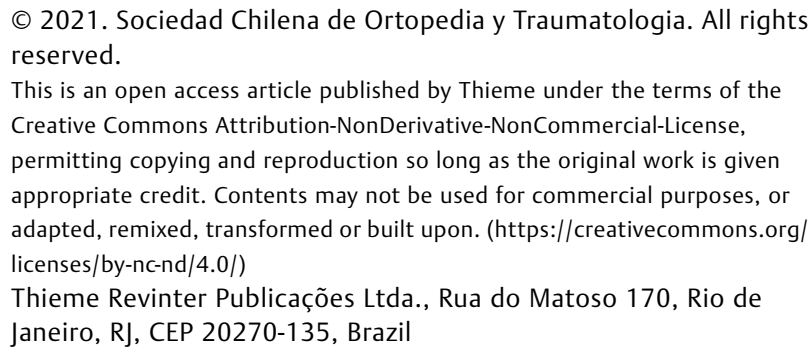

(c) 2021. Sociedad Chilena de Ortopedia y Traumatologia. All rights reserved.

This is an open access article published by Thieme under the terms of the Creative Commons Attribution-NonDerivative-NonCommercial-License, permitting copying and reproduction so long as the original work is given appropriate credit. Contents may not be used for commercial purposes, or adapted, remixed, transformed or built upon. (https://creativecommons.org/ licenses/by-nc-nd/4.0/)

Thieme Revinter Publicações Ltda., Rua do Matoso 170, Rio de Janeiro, RJ, CEP 20270-135, Brazil 


\begin{abstract}
Keywords

- medial patellofemoral ligament

- reconstruction

- patellar instability

Introduction Recurrent patellar instability can lead to poor functional results and progressive articular damage in the long term, and is frequently addressed by medial patellofemoral ligament reconstruction (MPFLR), with multiple techniques that most commonly differ regarding the method of patellar fixation.

Objective To evaluate the results of MPFLR using a novel technique of patellar fixation using transosseous sutures. The main objective is to determine the redislocation rates and functional results. The secondary goals include an assessment of complications and of other related variables, and a comparison between isolated MPFLR and MPFLR associated to tibial tubercle osteotomy.

Methods A cross-sectional study of 34 patients who underwent MPFLR from 2013 to 2019 with a minimum of 12 months of follow-up. The reconstruction was performed by the same first surgeon with double-bundle gracilis autograft in all cases. Fixation of the graft to the medial aspect of the patella was performed with two independent transosseous fixation points with high resistance sutures, and anatomic femoral fixation with an interference screw using anatomical and radiological landmarks. There were 27 patients with isolated MPFR, and 7 with associated tibial tubercle osteotomy.

Results The mean age was of 22.8 years (standard deviation [SD]: 9.1). Men comprised $50 \%$ of the sample. The mean follow-up from surgery to the application of the questionnaire was of 30.4 months (range: 12 to 72 months). The mean Kujala score at follow-up was of 89.4 (SD: 12.8; median: 93.5; range: 51 to 100). There were no cases of redislocation. No other complications were identified during the follow-up. In total, $81 \%$ of patients returned to sports, with $47 \%$ returning to their previous level of participation. No significant differences were found when comparing isolated MPFLR with MPFLR associated osteotomy groups.

Conclusion The MPFLR procedure using transosseous patellar fixation showed that patellar stability was restored in the short term to the midterm. This technique is safe and has excellent functional outcomes, and it prevents potential complications of patellar tunnels or the morbidity associated to the use of implants.
\end{abstract}

(rango: 12 a 72 meses). La puntuación media de Kujala en el seguimiento fue de 89,4 (DE: 12,8; mediana: 93,5; rango: 51 a 100). No hubo casos de reluxación. No se identificaron otras complicaciones durante el seguimiento. El $81 \%$ de los pacientes regresó a los deportes, y el $47 \%$ regresó a su nivel previo de participación. No se encontraron diferencias significativas al comparar RLPFM aislada con grupos de osteotomía asociada.

Conclusión La RLPFM mediante sutura transósea para fijación en la rótula mostró que la estabilidad rotuliana fue restaurada en el corto y mediano plazo. Esta técnica es segura, tiene excelentes resultados funcionales, y evita posibles complicaciones de los túneles rotulianos o morbilidad asociada al uso de implantes.

\section{Introducción}

Diferentes estructuras son responsables de la estabilidad rotuliana, incluidos factores musculares, ligamentosos, y óseos. Entre ellos, el complejo patelofemoral medial tiene un papel importante. El principal estabilizador medial de este complejo es el ligamento patelofemoral medial (LPFM). Los estudios biomecánicos ${ }^{1-3}$ muestran que contribuye del $50 \%$ al $80 \%$ a la contención medial de la rótula, y varía según el grado de flexión de la rodilla.
El desgarro del LPFM es un hallazgo común en las luxaciones agudas de la rótula, y es un factor importante que contribuye a la inestabilidad rotuliana recurrente, que puede conducir a peores resultados funcionales $\mathrm{y}$ posiblemente osteoartritis a largo plazo. ${ }^{1,4-7}$ La luxación patelar es más frecuente en los jóvenes, mujeres y pacientes que sufrieron una luxación previa. El riesgo de reluxación es del $17 \%$ tras el primer episodio, y del $50 \%$ tras el segundo, con alta variabilidad según la presencia de factores asociados. ${ }^{2,8-10}$ 
Factores anatómicos como la displasia troclear, la rótula alta, la lateralización de la tuberosidad tibial, y la inclinación patelar se consideran factores de riesgo importantes de recurrencia. ${ }^{11}$ El primer episodio de luxación se suele tratar de manera conservadora, pero en las luxaciones recurrentes se recomienda el tratamiento quirúrgico. ${ }^{12}$ Probablemente, la técnica quirúrgica más utilizada es la reconstrucción patelofemoral medial (RLPFM). ${ }^{13-15}$

Se ha descrito una gran variedad de técnicas para la reconstrucción patelofemoral medial, pero ninguna de ellas podría considerarse realmente un "gold-standard". 8 Las técnicas difieren en los tipos de injerto, sistema de fijación, presencia de túneles, tensionamiento y uso de implantes o dispositivos. Se han descrito diferentes opciones de injertos, pero nadie ha reportado ventajas significativas con respecto a los demás. ${ }^{13}$ El autoinjerto de isquiotibiales es probablemente el más utilizado para la reconstrucción de LPFM. ${ }^{12}$ Las técnicas de fijación de la rótula comunes para RLPFM incluyen anclas, túneles óseos, y tornillos interferenciales. $^{16-19}$ Los estudios que comparan los métodos de fijación muestran un mayor riesgo de fractura rotuliana en las técnicas de túneles óseos. ${ }^{17,20-25}$ También se han descrito suturas transóseas para RLPFM con reconstrucción de banda simple, ${ }^{26}$ pero no se encontraron estudios clínicos para reconstrucción de doble banda.

El objetivo de este estudio es evaluar las reconstrucciones de LPFM de doble banda en pacientes con luxación lateral de rótula recurrente intervenidos de una técnica novedosa de fijación de injerto rotuliano mediante suturas transóseas. El objetivo principal es determinar la tasa de reluxación y los resultados funcionales, incluido el regreso a los deportes, y el secundario es la evaluación de los factores de riesgo, de las lesiones asociadas, y de las complicaciones.

\section{Métodos}

Diseñamos un estudio observacional transversal que incluyó a pacientes que fueron sometidos a RLPFM con suturas transóseas patelares entre 2013 y 2019. Los criterios de inclusión fueron los siguientes: pacientes con luxación lateral de rótula recurrente, cirugía primaria de reconstrucción, uso de autoinjerto de isquiotibiales, fijación rotuliana con suturas transóseas, mínimo de 12 meses para la evaluación, y cirugía realizada por el mismo primer cirujano. Los datos obtenidos de las historias clínicas y los protocolos de los procedimientos incluyen la edad, la fecha de la lesión, la fecha de la cirugía, la evaluación clínica relevante en el momento de la presentación y en el seguimiento final, la presencia de factores de riesgo y de lesiones asociadas, y el estado meniscal y condral de la rodilla. La puntuación de Kujala postoperatoria y los datos relacionados con el retorno deportivo se obtuvieron de forma prospectiva a través de un cuestionario. ${ }^{27,28}$

La evaluación preoperatoria de rutina incluyó radiografías estándar, tomografía computarizada (TC), y resonancia magnética. Los factores de riesgo anatómicos se registraron utilizando el índice de Caton para la altura rotuliana en radiografías, la clasificación de Dejour para el grado de displasia, ${ }^{29}$ la distancia entre el tubérculo tibial y el surco troclear (TT-ST) en la TC para la lateralización del tubérculo tibial, y el ángulo de cadera-rodilla para la rotación femoral.

Todos los pacientes fueron intervenidos con una técnica quirúrgica estandarizada. Se coloca al paciente en decúbito supino sobre la mesa de operaciones. En todos los casos, se utiliza equipo de artroscopia estándar e intensificador de imágenes para radioscópica intraoperatoria. Primero, examen bajo anestesia para evaluar la laxitud de la rótula, prueba de desplazamiento lateral, medida en cuadrantes tanto en extensión completa como en $30^{\circ}$ y $60^{\circ}$ de flexión de rodilla. Luego, portales artroscópicos estándar para evaluar el estado del LPFM, lesiones condrales de la rótula, tracking dinámico patelofemoral, y detección y tratamiento de lesiones asociadas como las lesiones meniscales o condrales y los cuerpos libres. La extracción del autoinjerto de tendón gracilis se realiza de una manera mínimamente invasiva, mediante una incisión de $2 \mathrm{~cm}$ sobre la porción medial del pliegue posterior de flexión de la rodilla en $90^{\circ} \mathrm{de}$ flexión y abducción de la cadera, con el uso inicialmente de un stripper abierto para extraer la porción proximal de forma retrógrada, y luego un stripper cerrado para la parte distal del tendón de forma anterógrada. En pacientes en los que se planifica una osteotomía de la tuberosidad tibial tibial como procedimiento asociado, el tendón del gracilis se extrae de forma retrógrada, como se usa comúnmente, a través de la incisión del abordaje de osteotomía.

A continuación, abordamos la rótula con una incisión quirúrgica longitudinal sobre su borde medial superior. La incisión se profundiza hasta el hueso con electrocauterio, siempre de manera extraarticular, creando un lecho óseo que va desde el punto medio hasta la parte más proximal del borde medial de la rótula ( - Figura 1A). Posteriormente, con una broca de $1,5 \mathrm{~mm}$, se realizan 2 perforaciones oblicuas entre el borde más profundo (posterior) del lecho óseo y la cara anterior de la rótula con una inclinación de $45^{\circ}$, que sirven de paso a las suturas ( - Figura 1B). Una vez atravesado el hueso, las suturas se regresan por debajo del periostio para finalmente salir por el borde superficial (anterior) del lecho óseo descrito anteriormente. El procedimiento se repite a $2 \mathrm{~cm}$ a $3 \mathrm{~cm}$ de distancia. Por lo tanto, se obtienen dos puntos de anclaje para fijar el injerto en los tercios medio y proximal del borde medial de la rótula con suturas no absorbibles de alta resistencia (-Figure $\mathbf{1 C}$ ).

Una vez que el injerto se fija a la rótula, ambos extremos sueltos del injerto se apuntan al sitio femoral de fijación, y se suturan juntos cuidando de mantener la misma tensión en ambas riendas. El sitio femoral de fijación se aborda a través de una incisión cutánea transversal, y se identifica mediante palpación la fosa entre el epicóndilo medial y el tubérculo aductor. Este punto se revisa bajo radioscopía intraoperatoria en el punto anatómico descrito por Schöttle et al., ${ }^{30}$ teniendo especial cuidado en obtener una proyección lateral verdadera y adicionalmente se evalua su funcionalidad, probando la correcta isometría, comprobando la tensión del injerto en flexión y extensión, con el punto correcto detectado por mayor tensión en extensión completa. En ese punto, se coloca la guía de sobre la 


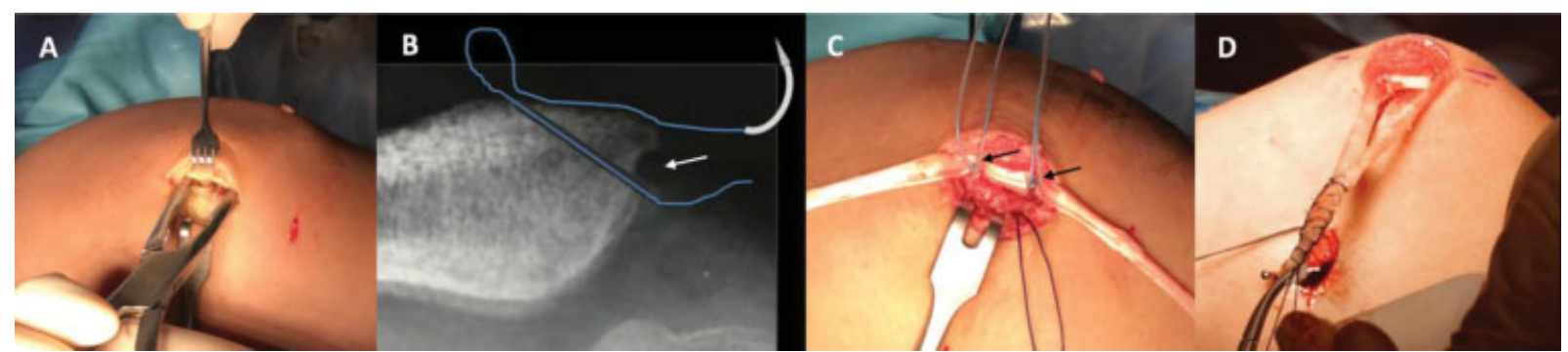

Fig. 1 (A) Preparación del lecho óseo en el lado medial de la rótula. (B) Radiografía axial de rótula con dibujo esquemático de perforación oblicua en $45^{\circ}$ y paso subperióstico de suturas de alta resistencia, que sirven como punto de anclaje en el lecho óseo (flecha blanca). (C) Autoinjerto de gracilis colocado sobre lecho óseo fijado con suturas de alta resistencia (suturas azules celestes) en la cara medial de la rótula, a través de ambos puntos de anclaje (flechas negras). (D) Injerto fijado en rótula listo para comprobar isometría de la fijación femoral.

cual se realiza un túnel de $7 \mathrm{~mm}$ y $40 \mathrm{~mm}$ de longitud (- Figura 1D). ${ }^{30}$ Se pasa el injerto entre las capas segunda (vasto medial) y tercera (cápsula) del complejo del ligamento patelofemoral medial, y se fija el fémur a la rodilla en aproximadamente $30^{\circ}$ de flexión con un tornillo interferencial de $7 \mathrm{~mm}$ x $28 \mathrm{~mm}$ y manteniendo el posicionamiento manual de la rótula para evitar la sobretensión. En los casos de fisis abierta, la fijación femoral se realizó con suturas alrededor de la inserción del tendón aductor distal.

Se realizó cirugía adicional para el manejo de los factores de riesgo asociados en los casos con altura rotuliana anormal significativa o lateralización de la tuberosidad tibial. Ni el grado de displasia ni el grado de versión femoral modificaron el protocolo. Se realizó una osteotomía tibial con medialización del tubérculo tibial en pacientes con índice TT-ST mayor de $20 \mathrm{~mm}$ y/o distalización del tubérculo tibial en los casos con índice de Caton de 1,4 o superior. Cuando se indicaron osteotomías con distalización, la medialización asociada se agregó sólo si el TT-ST era superior a $24 \mathrm{~mm}$, debido al "efecto medializante" intrínseco de la distalización. En los casos con fisis abierta, se realizó RLPFM aislada independientemente de las anomalías óseas para evitar alteraciones del crecimiento.

El análisis estadístico se realizó con las pruebas de chicuadrado y exacta de Fisher para variables paramétricas. Se utilizó la prueba mediana de Mood o de Mann-Whitney para las variables no paramétricas. La distribución normal de diferentes variables se probó con la prueba de AndersonDarling o Kolmogorov-Smirnov. Se utilizó la mediana para determinar diferencias en el caso de variables que no tuvieran distribución normal. El intervalo de confianza (IC) de las proporciones se calculó utilizando el método exacto para las proporciones. La significación estadística se definió para valores de $p$ inferiores a $5 \%$.

\section{Resultados}

El estudio incluyó inicialmente a 44 pacientes que cumplían los criterios, pero 10 pacientes no fueron accesibles en la evaluación final, por lo que finalmente se incluyó un total de 34 pacientes.

En este grupo de 34 pacientes, la edad media era de 22,8 años en el momento de la cirugía (rango: 10-42 años;
Tabla 1 Descripción de la serie de diferentes deportes practicados por los participantes $(n=34)$

\begin{tabular}{|l|l|}
\hline Variable & Resultados \\
\hline Fútbol & 15 \\
\hline Gimnasia & 5 \\
\hline Baloncesto & 5 \\
\hline Ciclismo & 4 \\
\hline Más de 1 deporte & 8 \\
\hline Otros deportes: & 12 \\
\hline $\begin{array}{l}\text { Voleibol, patinaje, pol dance, } \\
\text { danza, porrista, crossfit, } \\
\text { kickboxing, jogging, squash, } \\
\text { caminar, y levantamiento de pesas }\end{array}$ & \\
\hline Sin actividad física & 2 \\
\hline
\end{tabular}

desviación estándar [DE]: 9,1; mediana: 20 años). En cuanto al género, el 50\% (17 casos) fueron hombres, y 17 casos fueron mujeres.

La participación en deportes de nuestros pacientes fue de un $94 \%$ (solo 2 casos no realizan deportes), y se resume en la - Tabla 1. La evaluación clínica de estos pacientes mostró hiperlaxitud articular generalizada en 8 (24\%), genu valgo en $14(42 \%)$, y presencia de inestabilidad clínica significativa con signo de la "J" positivo en 2 (6\%). De acuerdo a los factores anatómicos estudiados en nuestro protocolo, la displasia troclear fue el hallazgo más frecuente en 30 casos (88\%). El grado de displasia según el sistema de clasificación de Dejour se presenta en la - Tabla 2 . La rótula alta según el índice de Caton mayor de 1,2 se encontró en 8 pacientes (24\%), con 2 (6\%) de ellos con un índice de 1,4. Ningún paciente presentó Caton mayor que 1,4 .

Se encontró lateralización anormal del tubérculo tibial según la distancia TT-ST (más de $15 \mathrm{~mm}$ ) en 24 pacientes (71\%). De estos pacientes, 11 tenían más de $20 \mathrm{~mm}$, y se consideraron para corrección quirúrgica mediante osteotomía con medialización del tubérculo tibial. En total, 4 pacientes de este grupo tenían fisis abierta, lo que contraindicó el procedimiento; por eso, se realizó osteotomía en los 7 pacientes restantes. De estos 7 
Tabla 2 Descripción de la serie de displasia según la clasificación de Dejour $(\mathrm{n}=34)$

\begin{tabular}{|l|l|}
\hline Variable & Resultados \\
\hline Displasia: & $30(88 \%)$ \\
\hline Dejour A & 16 \\
\hline Dejour B & 9 \\
\hline Dejour C & 4 \\
\hline Dejour D & 1 \\
\hline Sin displasia & $4(12 \%)$ \\
\hline
\end{tabular}

pacientes, 2 tenían un índice de Caton concomitante de 1,4, por lo que la osteotomía se realizó con un efecto de distalización asociado a medialización (-Tabla 3 ).

Durante la artroscopia inicial, el examen sistemático de rutina de toda la articulación en busca de lesiones asociadas y cuerpos libres mostró una alta prevalencia de lesiones condrales, significativamente más frecuentes en la rótula (31 casos, 91\%). Sólo se encontraron lesiones menores los meniscos, que no requirieron tratamiento específico, y 12 pacientes (35\%) tenían cuerpos libres que fueron removidos (-Tabla 4).

El tiempo medio transcurrido entre la primera luxación y la cirugía fue de 72 meses (rango: 1 a 328 meses; DE: 85,4; mediana: 39,7). El seguimiento promedio desde la cirugía a la evaluación postquirúrgica con cuestionario fue de 30,4 meses (DE: 14,6) con un rango de 12 a 72 meses.

En cuanto a los resultados funcionales, la media de Kujala fue de 89,4 puntos (DE: 12,8), con mediana de 93,5 y rango de 51 a 100 . No hubo casos de luxación postoperatoria durante el periodo de seguimiento. No se registró ninguna cirugía de revisión. Solo 2 pacientes (6\%) se quejaron de dolor postoperatorio o inestabilidad leve durante el deporte o las
Tabla 4 Hallazgos artroscópicos en 34 pacientes

\begin{tabular}{|l|l|}
\hline Variable & Resultados \\
\hline Lesiones meniscales & $7(21 \%)$ \\
\hline Lesiones condrales: & \\
\hline Fémoro-tibial medial & 0 \\
\hline Fémoro-tibial lateral & $5(15 \%)$ \\
\hline Femoropatelar: & $32(94 \%)$ \\
\hline Sin lesiones & $2(6 \%)$ \\
\hline Troclear aislada & $1(3 \%)$ \\
\hline Rotuliana aislada & $25(73 \%)$ \\
\hline Ambas & $6(18 \%)$ \\
\hline Cuerpo suelto & $12(35 \%)$ \\
\hline
\end{tabular}

actividades diarias. No se identificaron otras complicaciones durante el seguimiento.

Con respecto a la tasa de retorno a los deportes, de los pacientes que participaron en deportes antes de la operación (32/34 pacientes), el $81 \%$ (26 casos) regresaron a los deportes después de la reconstrucción del LPFM, y el 47\% (15 casos), a su nivel deportivo previo. El tiempo medio para volver a los deportes fue de 8,8 meses (DE: 5,5 meses), con una mediana de 6,5, y un rango de 2 a 24 .

Se compararon los dos grupos de acuerdo a nuestro protocolo de tratamiento según la indicación de ostetomía de tuberosidad tibial: uno tratado con RLPFM aislada (27 pacientes) y otro con RLPFM asociada a una osteotomía (7 pacientes, 21\%). Al comparar estos dos grupos, no encontramos diferencias con respecto a la puntuación de Kujala, la tasa de dislocación, o la tasa de retorno a los deportes, como se ve en la - Tabla $\mathbf{5}$.

Tabla 3 Casos de pacientes con TT-ST $>20 \mathrm{~mm}(\mathrm{n}=11)$

\begin{tabular}{|c|c|c|c|c|c|}
\hline Género & Edad & TT-ST & $\begin{array}{l}\text { Índice } \\
\text { de Caton }\end{array}$ & Displasia* & OTT asociada \\
\hline Mujer & 14 & $24 \mathrm{~mm}$ & 1,2 & $A$ & No (fisis abierta) \\
\hline Mujer & 13 & $22 \mathrm{~mm}$ & 1,1 & A & No (fisis abierta) \\
\hline Hombre & 14 & $25 \mathrm{~mm}$ & 1,3 & A & No (fisis abierta) \\
\hline Hombre & 35 & $21 \mathrm{~mm}$ & 1,1 & A & No (fisis abierta) \\
\hline Mujer & 20 & $23 \mathrm{~mm}$ & 1,1 & $\mathrm{C}$ & Medialización \\
\hline Mujer & 20 & $22 \mathrm{~mm}$ & 1,1 & $\mathrm{C}$ & Medialización \\
\hline Mujer & 27 & $23 \mathrm{~mm}$ & 1,1 & A & Medialización \\
\hline Mujer & 17 & $23 \mathrm{~mm}$ & 1,2 & B & Medialización \\
\hline Hombre & 39 & $23 \mathrm{~mm}$ & 1,2 & C & Medialización \\
\hline Hombre & 15 & $25 \mathrm{~mm}$ & 1,4 & B & Medial + distalización \\
\hline Hombre & 15 & $24 \mathrm{~mm}$ & 1,4 & $\mathrm{C}$ & Medial + distalización \\
\hline
\end{tabular}

Nota: *Según la clasificación de Dejour de displasia patelofemoral.

Abreviaturas: OTT, osteotomía del tubérculo tibial; TT-ST, distancia entre el tubérculo tibial y el surco troclear. 
Tabla 5 Comparación entre RLPFM aislada y RLPFM + OTT asociada

\begin{tabular}{|l|l|l|l|l|}
\hline Grupo & Tasa de reluxación & Kujala Mediana & Kujala Media & Tasa de regreso al deporte \\
\hline RLPFM aislada & $0 \%$ & 93 (IC: $89,9-95)$ & 88 (IC: $82.7-93,6)$ & $78 \%$ (IC: $58 \%-91 \%)$ \\
\hline RLPFM + OTT asociada & $0 \%$ & 96 (IC: $88,6-100)$ & 94 (IC: $89,1-99,4)$ & $71 \%$ (IC: $29 \%-96 \%)$ \\
\hline valor de $p$ & No aplica & 0,671 & 0,083 & 0,736 \\
\hline
\end{tabular}

Abreviaturas: IC, intervalo de confianza; OTT, osteotomía del tubérculo tibial; RLPFM, reconstrucción del ligamento patelofermoral medial.

\section{Discusión}

Los objetivos principales de este estudio fueron evaluar los resultados funcionales y las tasas de reluxación. En lo que se refiere a la evaluación de los resultados funcionales, la puntuación de Kujala es una de las escalas más utilizadas en las afecciones patelofemorales. ${ }^{1,28}$ Schneider et al., ${ }^{31}$ en su revisión sistemática, encontraron una estimación combinada entre 81,6 y 90 puntos utilizando la puntuación de Kujala. Además de esto, estimaron el riesgo de inestabilidad objetiva recurrente después de la cirugía entre $0,3 \%$ y 2,1\%. ${ }^{31}$ Resumimos, en el -Apéndice 1, los hallazgos comparativos en diferentes estudios clínicos que incluyeron más de 30 pacientes, principalmente obtenidos, de 2 revisiones sistemáticas importantes. ${ }^{1,47}$ Las tasas de reluxación entre ellas varían entre el $0 \%$ y el $4,5 \%$, y las puntuaciones de Kujala, entre 75 y 94, comparables a los hallazgos de nuestra serie.

En nuestra serie, la puntuación media de Kujala fue de 89,4 puntos, y no se encontraron episodios de luxación (0\%) en un seguimiento medio de 30 meses.

Lograr una adecuada estabilidad rotuliana con resultados funcionales satisfactorios es probablemente el principal objetivo de nuestra cirugía, pero el retorno al deporte es de crucial importancia al considerar nuestros resultados y las expectativas de los pacientes, especialmente en la población joven y activa. Sin embargo, pocos estudios reportan su tasa de retorno a los deportes como lo muestran Schneider et al. ${ }^{31}$ Ellos informan una estimación combinada de la tasa de retorno al deporte del 84\% (IC: 71\% a 97\%), comparable al 81\% de nuestro estudio. Al considerar a los pacientes que regresan a su nivel anterior de participación, los resultados son significativamente más bajos; Matassi et a. ${ }^{32}$ y Lippacher et al. ${ }^{19}$ reportan $39 \%$ y $53 \%$, respectivamente. No obstante, con un seguimiento más largo (6 años), Ambrožič y Novak ${ }^{33}$ reportan un $70 \%$ de retorno al nivel anterior. Muchos estudios ${ }^{34,35}$ que abordan este tema muestran una recuperación lenta con programas prolongados de rehabilitación (más de 8 meses) después de una reconstrucción del LPFM. Hay informes de recuperación aún más lenta si se la asocia a una osteotomía tibial. ${ }^{19}$ Se ha demostrado que déficits menores en la evaluación global pueden permanecer un largo período de tiempo después del alta para la práctica deportiva. ${ }^{36}$

Las técnicas de RLPFM han mostrado muchas diferencias entre los estudios clínicos. El tipo de fijación de la rótula es probablemente uno de los más significativos, con una gran variedad que dificulta la comparación.

En general, podemos dividir la fijación rotuliana en tres tipos: anclas, túneles óseos, y técnicas de sutura, como sugiere la revisión sistemática de Kang et al. ${ }^{37}$ Estos autores no encontraron diferencias entre los tres tipos en términos de la puntuación de Kujala, de las tasas de reluxación, o de las complicaciones. Por otro lado, muchos autores han encontrado más complicaciones relacionadas con el uso de túneles óseos en la rótula, especialmente fracturas rotulianas, y dolor persistente. ${ }^{17,20-25,38,39}$ Desai et al., ${ }^{1}$ en su revisión sistemática, mostraron tasas de fracturas rotulianas entre $0 \%$ y $17 \%$, significativamente mayor en las técnicas de túnel óseo, a pesar de que los resultados clínicos son similares a los de las técnicas de fijación cortical. El riesgo de fractura aumenta a medida que aumenta el tamaño del túnel, especialmente para aquellos mayores de $4,5 \mathrm{~mm}$, como se observa con las técnicas de Endobutton. No obstante, no se informaron fracturas con túneles menores que $3 \mathrm{~mm} .{ }^{1,25}$ Nuestra técnica aborda esta preocupación mediante el uso de una pequeña perforación oblicua de $1,5 \mathrm{~mm}$ sólo para el paso de agujas, y no se encontraron fracturas en el presente estudio.

La fijación con sutura transósea ha mostrado una fuerza de fijación adecuada en pruebas biomecánicas en modelos cadavéricos, y una carga máxima hasta el fallo (540 $\mathrm{N} \pm 160 \mathrm{~N}$ ) más fuerte que la del LPFM nativo y la de otros métodos de fijación (ancla, tornillo de interferencial, túneles óseos transpatelares o puente óseo medial). ${ }^{16,18}$

Por lo que sabemos, ningún estudio clínico ha informado de esta técnica ni los resultados clínicos de la reconstrucción de LPFM de doble banda con fijación transósea de la rótula.

Una ventaja notable de la técnica descrita en este estudio con suturas transóseas es su bajo costo, que evita implantes más costosos en rótula, como anclas, tornillos o dispositivos de suspensión.

Otras complicaciones de la RLPFM publicadas entre las revisiones sistemáticas son un signo de aprensión postoperatoria positivo entre 3,6\% y $8,2 \%$ y riesgo de reoperación de $3,1 \%$ a 4,2\%. ${ }^{25,31}$ Encontramos 2 casos (6\%) con signo de aprensión después de la cirugía, y no fue necesaria la cirugía de revisión.

La inestabilidad rotuliana implica una amplia variedad de anomalías anatómicas que hacen que la rótula sea propensa a luxarse, como la displasia troclear, la rótula alta, la distancia TT-ST, y la inclinación patelar, que se han considerado importantes factores de riesgo de recurrencia. ${ }^{11,29}$ La literatura actual respalda procedimientos asociados en pacientes con factores de riesgo importantes demostrados y luxación lateral recurrente, como el signo de la "J" con displasia troclear grave, lateralización de la tuberosidad tibial con TT-ST superior a $20 \mathrm{~mm}$, rótula alta con índice de Caton superior a 1,2 a 1,4, y anomalías graves de la alineación 
(genu valgo grave o anteversión femoral superior a $\left.30^{\circ}\right) .40-44$ En este contexto, la transferencia de tuberosidad tibial tiene su función cuando se considera la corrección de anomalías como la rótula alta o una TT-ST excesiva. Magnussen et al., ${ }^{44}$ en su revisión sistemática sobre la distalización del tubérculo tibial en pacientes con rótula alta, informan que este procedimiento es seguro y reduce eficazmente la luxación rotuliana recurrente con un riesgo general de reluxación de casi el $2 \%$, con resultados funcionales de buenos a excelentes. ${ }^{44} \mathrm{Al}$ considerar la lateralización de TT, en una revisión sistemática, Boutefnouchet et al. ${ }^{43}$ informaron que la literatura es escasa y heterogénea, y que no se pudo determinar valores que definan la necesidad de la indicación quirúrgica, por ejemplo del TT-ST. En 1994, Dejour et al. ${ }^{11}$ utilizaron un punto de corte de $20 \mathrm{~mm}$; sin embargo, el $20 \%$ de las rodillas asintomáticas excedió ese valor. Esto es similar a lo informado por Caplan et al.; ${ }^{45}$ por lo tanto, con la evidencia actual, la medición de TT-ST debe usarse con precaución y en combinación con otros hallazgos para decidir si la corrección quirúrgica es necesaria. ${ }^{11,43,45}$

Mulliez et al., ${ }^{46}$ en un estudio con 129 pacientes, no encontraron diferencias al comparar los resultados funcionales entre RLPFM aislada (91 pacientes, 70\%) y asociada a osteotomía tibial (38 pacientes del grupo, 30\%) en pacientes con TT-ST mayor que $20 \mathrm{~mm}$ e índice de Caton mayor que $1,2 .{ }^{46}$ Según nuestro protocolo, 11 de nuestros pacientes (32\%) tenían indicación de osteotomía, pero, por presentar fisis abierta en 4 de ellos, realizamos una osteotomía asociada en 7 casos, y no encontramos diferencias en resultados funcionales, luxaciones o complicaciones. Debemos ser conscientes de que estos dos grupos no son del todo comparables debido al mayor grado de factores anatómicos anormales en el grupo de osteotomía, pero aún así los resultados no mostraron diferencias significativas.

Las limitaciones del estudio son las siguientes: tamaño de muestra pequeño (34 pacientes), que puede afectar la comparación de grupos, y se requiere un seguimiento más prolongado para evaluar la eficacia a largo plazo de esta técnica, aunque la mayoría de los estudios encontrados en la literatura comparten ambos problemas. Otra limitación son los grupos heterogéneos de pacientes con diferentes grados de anomalías óseas y con procedimientos asociados, que podrían limitar la generalización de la técnica. Finalmente, la tasa de pacientes no accesibles fue del $23 \%$, lo que es comparable a lo encontrado en la literatura. 1,31,47

\section{Conclusión}

La reconstrucción del ligamento patelofemoral medial mediante fijación rotuliana con suturas transóseas mostró excelentes resultados funcionales, con una tasa aceptable de retorno a la actividad deportiva y sin casos de reluxación con un seguimiento de 1 a 6 años. La técnica quirúrgica descrita es segura, y podría evitar posibles complicaciones relacionadas con el uso de túneles rotulianos y a también morbilidad y costos adicionales relacionados con el uso de implantes.

\section{Conflicto of Intereses}

Los autores no tienen conflicto de intereses que declarar.

\section{Referencias}

1 Desai VS, Tagliero AJ, Parkes CW, et al. Systematic Review of Medial Patellofemoral Ligament Reconstruction Techniques: Comparison of Patellar Bone Socket and Cortical Surface Fixation Techniques. Arthroscopy 2019;35(05):1618-1628

2 Krebs C, Tranovich M, Andrews K, Ebraheim N. The medial patellofemoral ligament: Review of the literature. J Orthop 2018;15(02):596-599

3 Gonçaives MB, Júnior LH, Soares LF, Gonçaives TJ, Dos Santos RL, Pereira ML. Medial patellofemoral ligament reconstruction to treat recurrent patellar dislocation. Rev Bras Ortop 2015;46 (02):160-164

4 Atkin DM, Fithian DC, Marangi KS, Stone ML, Dobson BE, Mendelsohn C. Characteristics of patients with primary acute lateral patellar dislocation and their recovery within the first 6 months of injury. Am J Sports Med 2000;28(04):472-479

5 Mäenpää H, Lehto MU. Patellofemoral osteoarthritis after patellar dislocation. Clin Orthop Relat Res 1997;(339):156-162

6 Sillanpää P, Mattila VM, Iivonen T, Visuri T, Pihlajamäki H. Incidence and risk factors of acute traumatic primary patellar dislocation. Med Sci Sports Exerc 2008;40(04):606-611

7 Sillanpää PJ, Mattila VM, Visuri T, Mäenpää H, Pihlajamäki H. Patellofemoral osteoarthritis in patients with operative treatment for patellar dislocation: a magnetic resonance-based analysis. Knee Surg Sports Traumatol Arthrosc 2011;19(02): 230-235

8 Blønd L. Patellar Instability - An Update. Acta Orthop Belg 2017;83 (03):367-386

9 Fithian DC, Paxton EW, Stone ML, et al. Epidemiology and natural history of acute patellar dislocation. Am J Sports Med 2004;32 (05):1114-1121

10 Subramanian P, Patel R. Patellofemoral instability: an overview. Orthop Trauma 2019;33(02):119-126

11 Dejour H, Walch G, Nove-Josserand L, Guier C. Factors of patellar instability: an anatomic radiographic study. Knee Surg Sports Traumatol Arthrosc 1994;2(01):19-26

12 Stefancin JJ, Parker RD. First-time traumatic patellar dislocation: a systematic review. Clin Orthop Relat Res 2007;455(455):93-101

13 Amin NH, Lynch TS, Patel RM, Patel N, Saluan P. Medial Patellofemoral Ligament Reconstruction. JBJS Rev 2015;3(07):3-4

14 Howells NR, Barnett AJ, Ahearn N, Ansari A, Eldridge JD. Medial patellofemoral ligament reconstruction: a prospective outcome assessment of a large single centre series. J Bone Joint Surg $\mathrm{Br}$ 2012;94(09):1202-1208

15 Mistry JB, Bonner KF, Gwam CU, Thomas M, Etcheson JI, Delanois RE. Management of Injuries to the Medial Patellofemoral Ligament: A Review. J Knee Surg 2018;31(05):439-447

16 Russ SD, Tompkins M, Nuckley D, Macalena J. Biomechanical comparison of patellar fixation techniques in medial patellofemoral ligament reconstruction. Am J Sports Med 2015; 43(01):195-199

17 Migliorini F, Driessen A, Quack V, Schenker H, Tingart M, Eschweiler J. Patellar fixation graft via suture anchors versus tunnel techniques during isolated MPFL reconstruction for recurrent patellofemoral instability: a systematic review of the literature. Arch Orthop Trauma Surg 2020;140(09): 1201-1210

18 Lenschow S, Schliemann B, Gestring J, Herbort M, Schulze M, Kösters C. Medial patellofemoral ligament reconstruction: fixation strength of 5 different techniques for graft fixation at the patella. Arthroscopy 2013;29(04):766-773

19 Lippacher S, Dreyhaupt J, Williams SR, Reichel H, Nelitz M. Reconstruction of the medial patellofemoral ligament: clinical 
outcomes and return to sports. Am J Sports Med 2014;42(07): 1661-1668

20 Lippacher S, Reichel H, Nelitz M. [Patellar fracture after patellar stabilization]. Orthopade 2010;39(05):516-518

21 Parikh SN, Nathan ST, Wall EJ, Eismann EA. Complications of medial patellofemoral ligament reconstruction in young patients. Am J Sports Med 2013;41(05):1030-1038

22 Parikh SN, Wall EJ. Patellar fracture after medial patellofemoral ligament surgery: a report of five cases. J Bone Joint Surg Am 2011;93(17):e97-(1-8)

23 Sanchis-Alfonso V, Montesinos-Berry E, Ramirez-Fuentes C, LealBlanquet J, Gelber PE, Monllau JC. Failed medial patellofemoral ligament reconstruction: Causes and surgical strategies. World J Orthop 2017;8(02):115-129

24 Schiphouwer L, Rood A, Tigchelaar S, Koëter S. Complications of medial patellofemoral ligament reconstruction using two transverse patellar tunnels. Knee Surg Sports Traumatol Arthrosc 2017;25(01):245-250

25 Shah JN, Howard JS, Flanigan DC, Brophy RH, Carey JL, Lattermann C. A systematic review of complications and failures associated with medial patellofemoral ligament reconstruction for recurrent patellar dislocation. Am J Sports Med 2012;40(08):1916-1923

26 Brown GD, Ahmad CS. The docking technique for medial patellofemoral ligament reconstruction. Oper Tech Orthop 2007;17(04): 216-222

27 Gil-Gámez J, Pecos-Martín D, Kujala UM, et al. Validation and cultural adaptation of "Kujala Score" in Spanish. Knee Surg Sports Traumatol Arthrosc 2016;24(09):2845-2853

28 Kujala UM, Jaakkola LH, Koskinen SK, Taimela S, Hurme M, Nelimarkka O. Scoring of patellofemoral disorders. Arthroscopy 1993;9(02):159-163

29 Dejour D, Le Coultre B. Osteotomies in patello-femoral instabilities. Sports Med Arthrosc Rev 2007;15(01):39-46

30 Schöttle PB, Schmeling A, Rosenstiel N, Weiler A. Radiographic landmarks for femoral tunnel placement in medial patellofemoral ligament reconstruction. Am J Sports Med 2007;35(05):801-804

31 Schneider DK, Grawe B, Magnussen RA, et al. Outcomes After Isolated Medial Patellofemoral Ligament Reconstruction for the Treatment of Recurrent Lateral Patellar Dislocations: A Systematic Review and Meta-analysis. Am J Sports Med 2016;44(11): 2993-3005

32 Matassi F, Innocenti M, Andrea C, Zanna L, Malone J, Civinini R. Timing for Safe Return to Sport after Medial Patellofemoral Ligament Reconstruction: The Role of a Functional Test Battery. J Knee Surg. 2019 Sep 4

33 Ambrožič B, Novak S. The influence of medial patellofemoral ligament reconstruction on clinical results and sports activity level. Phys Sportsmed 2016;44(02):133-140

34 Krych AJ, O'Malley MP, Johnson NR, et al. Functional testing and return to sport following stabilization surgery for recurrent lateral patellar instability in competitive athletes. Knee Surg Sports Traumatol Arthrosc 2018;26(03):711-718
35 Saper MG, Fantozzi P, Bompadre V, Racicot M, Schmale GA. Return-to-Sport Testing After Medial Patellofemoral Ligament Reconstruction in Adolescent Athletes. Orthop J Sports Med 2019;7(03):232-235

36 Shams K, DiCesare CA, Grawe BM, et al. Biomechanical and Functional Outcomes After Medial Patellofemoral Ligament Reconstruction: A Pilot Study. Orthop J Sports Med 2019;7(02): 232-237

37 Kang H, Zheng R, Dai Y, Lu J, Wang F. Single- and double-bundle medial patellofemoral ligament reconstruction procedures result in similar recurrent dislocation rates and improvements in knee function: a systematic review. Knee Surg Sports Traumatol Arthrosc 2019;27(03):827-836

38 Christiansen SE, Jacobsen BW, Lund B, Lind M. Reconstruction of the medial patellofemoral ligament with gracilis tendon autograft in transverse patellar drill holes. Arthroscopy 2008;24(01):82-87

39 Dhinsa BS, Bhamra JS, James C, Dunnet W, Zahn H. Patella fracture after medial patellofemoral ligament reconstruction using suture anchors. Knee 2013;20(06):605-608

40 Carstensen SE, Menzer HM, Diduch DR. Patellar Instability: When is Trochleoplasty Necessary? Sports Med Arthrosc Rev 2017;25 (02):92-99

41 Balcarek P, Rehn S, Howells NR, et al. Results of medial patellofemoral ligament reconstruction compared with trochleoplasty plus individual extensor apparatus balancing in patellar instability caused by severe trochlear dysplasia: a systematic review and meta-analysis. Knee Surg Sports Traumatol Arthrosc 2017;25(12):3869-3877

42 Kita K, Tanaka Y, Toritsuka Y, et al. Factors Affecting the Outcomes of Double-Bundle Medial Patellofemoral Ligament Reconstruction for Recurrent Patellar Dislocations Evaluated by Multivariate Analysis. Am J Sports Med 2015;43(12):2988-2996

43 Boutefnouchet T, Downham C, Bassett J, Thompson P, Sprowson A. The Efficacy of Medial Patellofemoral Ligament Reconstruction Combined with Tibial Tuberosity Transfer in the Treatment of Patellofemoral Instability. Knee Surg Relat Res 2016;28(02): 99-109

44 Magnussen RA, De Simone V, Lustig S, Neyret P, Flanigan DC. Treatment of patella alta in patients with episodic patellar dislocation: a systematic review. Knee Surg Sports Traumatol Arthrosc 2014;22(10):2545-2550

45 Caplan N, Lees D, Newby M, et al. Is tibial tuberosity-trochlear groove distance an appropriate measure for the identification of knees with patellar instability? Knee Surg Sports Traumatol Arthrosc 2014;22(10):2377-2381

46 Mulliez A, Lambrecht D, Verbruggen D, Van Der Straeten C, Verdonk P, Victor J. Clinical outcome in MPFL reconstruction with and without tuberositas transposition. Knee Surg Sports Traumatol Arthrosc 2017;25(09):2708-2714

47 Yeung M, Leblanc MC, Ayeni OR, et al. Indications for Medial Patellofemoral Ligament Reconstruction: A Systematic Review. J Knee Surg 2016;29(07):543-554 
112 Reconstrucción del ligamento patelofemoral medial con suturas transóseas patelares Pakuts et al.

Apéndice 1 Estudios con más de 30 pacientes

\begin{tabular}{|c|c|c|c|c|c|}
\hline Estudio & $\begin{array}{l}\mathrm{N}^{\circ} \text { de } \\
\text { pacientes }\end{array}$ & $\begin{array}{l}\text { Seguimiento } \\
\text { medio (meses) }\end{array}$ & $\begin{array}{l}\text { Tipo } \\
\text { de injerto }\end{array}$ & $\begin{array}{l}\text { Puntuación Kujala } \\
\text { (desviación estándar)* }^{*}\end{array}$ & Reluxación \\
\hline Howells et al., $2012^{y}$ & 193 & 16 & ST & 81,7 & 0 \\
\hline Astur et al., $2015^{d}$ & 58 & 60 & GC & $79,6(14,5)$ & 0 \\
\hline Goyal et al., $2013^{d}$ & 32 & 38 & $\mathrm{CD}$ & 91,3 & 0 \\
\hline Kang et al., $2014^{d}$ & 45 & 34 & ST & $90,9(6,6)$ & 0 \\
\hline Kita et al., $2015^{d}$ & 42 & 38 & ST & 93,6 & $4,5 \%$ \\
\hline Krishna Kumar et al., $2014^{\mathrm{d}}$ & 30 & 25 & GC & 87 & 0 \\
\hline Lee et al., $2017^{d}$ & 44 & 48 & GC/sintético & $83(16)$ & 0 \\
\hline Mulliez et al., $2015^{\mathrm{d}}$ & 124 & 12 & GC/ST & $74,7(20,5)$ & $0,8 \%$ \\
\hline Niu et al., $2017^{d}$ & 64 & 25 & ST & $91,8(3,7)$ & 0 \\
\hline Niu et al., $2017^{d}$ & 32 & 48 & ST & $92(4,8)$ & 0 \\
\hline Panni et al., $2011^{d}$ & 51 & 33 & ST & $86,8(14,4)$ & 0 \\
\hline Valkering et al., $2017^{\mathrm{d}}$ & 31 & 37 & GC & $80,9(2)$ & 0 \\
\hline Von Engelhardt et al., $2018^{d}$ & 30 & 24 & GC & $92(10)$ & 0 \\
\hline Wagner et al., $2013^{d}$ & 50 & $12-24$ & GC & $87(13)$ & $2 \%$ \\
\hline Han et al., $2011^{y}$ & 52 & 68 & ST & 82,6 & 0 \\
\hline Lippacher et al., $2014^{y}$ & 68 & 25 & GC & 87,5 & $2 \%$ \\
\hline Ma et al., $2013^{y}$ & 32 & 40 & ST & 87 & 0 \\
\hline Matsushita et al., $2014^{y}$ & 34 & 108 & ST & 92 & 0 \\
\hline Panni et al., $2011^{y}$ & 48 & 33 & ST & $86,8(7,2)$ & 0 \\
\hline Smith et al., $2014^{y}$ & 30 & 12 & ST/GC & $84,1(20,6)$ & $1 \%$ \\
\hline Wang et al., $2013^{y}$ & 58 & 48 & ST & $92,9(4,32)$ & 0 \\
\hline Zhou et al., $2014^{y}$ & 32 & 18 & ST & $91(7)$ & 0 \\
\hline Presente estudio & 34 & 30 & GC & $89,4(12,8)$ & 0 \\
\hline
\end{tabular}

Abreviaturas: GC, gracilis; CD, cuádriceps; ST, semitendinoso; y

Notas: *Si está disponible; ${ }^{y}$ revisión sistémica de Yeung et al. ${ }^{47} ;{ }^{\text {d }}$ revisión sistemática de Desai et al. ${ }^{1}$ 